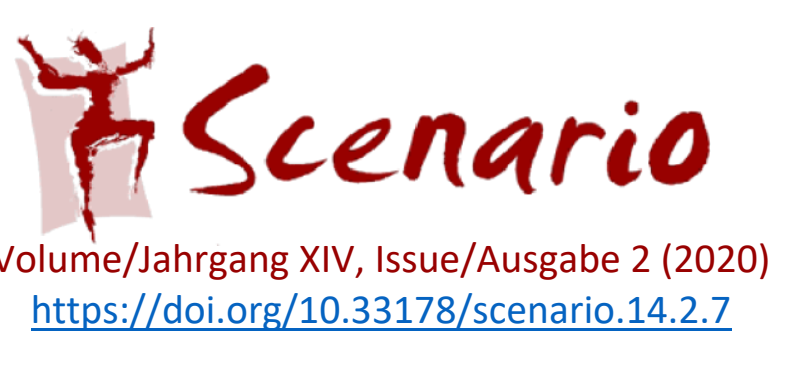

\title{
To Zoom or not to Zoom: Adapting Educational Versions of Shakespeare's Plays for an Online Platform During the Covid Pandemic
}

\author{
Peadar Donohoe, Marcus Bale
}

In this contribution to the Window of Practice-section, we will introduce the reader to Cyclone Rep's interactive Shakespeare plays for secondary school students and then discuss the challenges we faced adapting our work for the online medium.

\section{Introduction}

Through our theatre company, Cyclone Rep, we have been presenting educational versions of Shakespeare's plays at Irish venues and schools for secondary school students since 2005. From 2009 we adopted the moniker The Shakespeare Sessions to brand our multi-faceted approach to presenting Shakespeare's plays. Our approach to Shakespeare theatre is not a traditional one. To give you an idea of what that is, we'll begin the introduction with a picture from one of our productions of the Merchant of Venice.

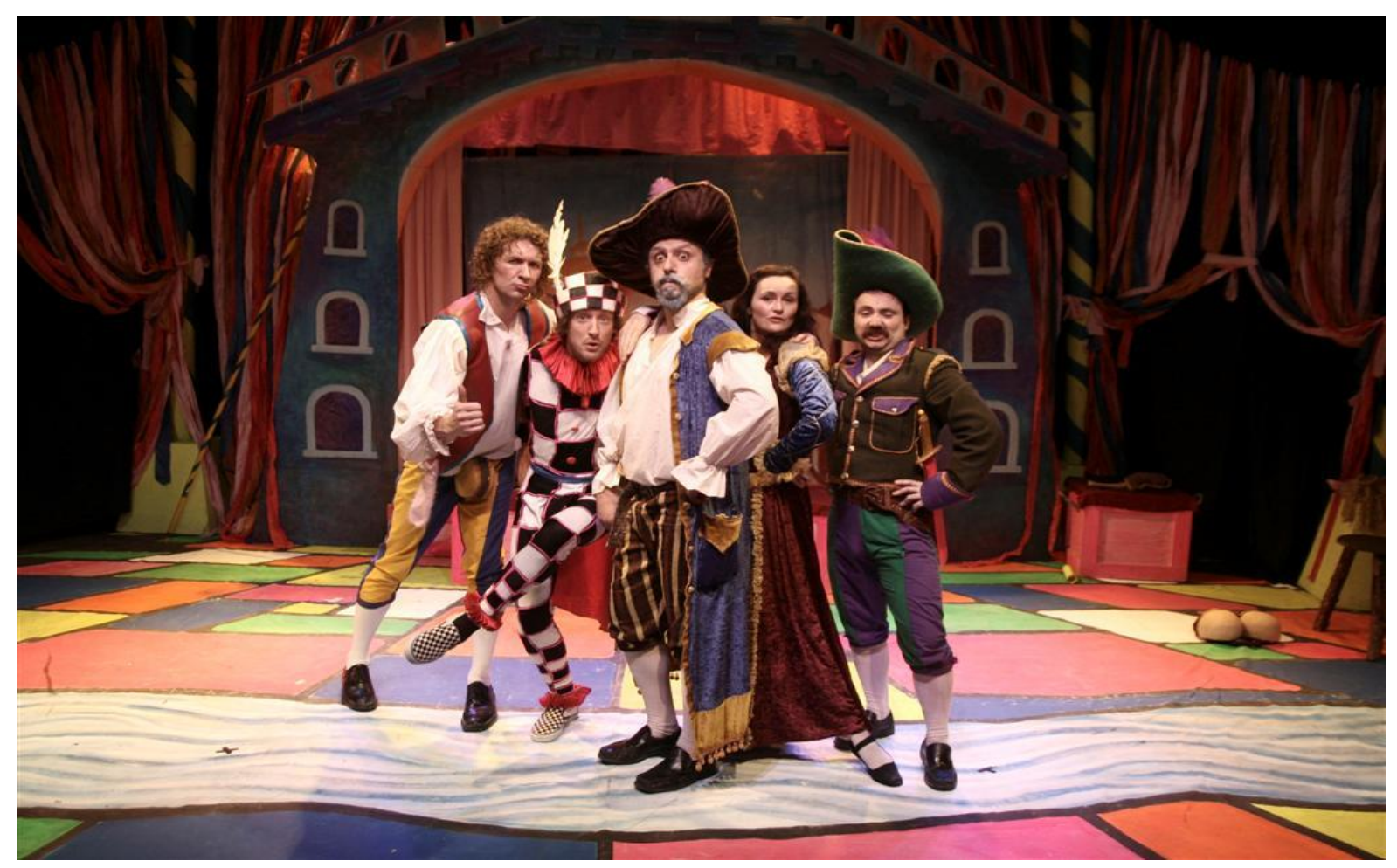

Fig. 1: A commedia dell'Arte inspired version of the Merchant of Venice, directed by Peadar Donohoe with actors from left: Martin Condon, Damian Punch, Marcus Bale, Paula McGlinchey and Sean McNally 
You may have gleaned from the picture above that our productions are humorous. They are produced this way in order to appeal to secondary school students who may never have experienced Shakespeare's plays or stepped inside a theatre before. However, humour is just one tool that we use to help bridge the gap in learner understanding of Shakespeare's language and poetry.

Another key tool is that our actors are directed to give heightened physical performances that include using their bodies and gestures to match Shakespeare's heightened language - thus helping to bring the imagery to life. This is crucial for audiences that are not used to the language of Shakespeare, as it can help them to understand the gist of what is being said, even when they may have little understanding of all the actual words. The choice to direct actors this way came from our experience attending productions of Shakespeare for schools where the actors just stood on the stage in one spot, delivering text, while losing the audience completely, as the students begin to talk amongst themselves and ignore the ensuing onstage performance. We did not want this to be a young audience's experience at our plays.

Other tools employed for learning and understanding are the use of audio visuals and animations to highlight themes and plot developments, hot-seating characters to explore their motivations, breaking the fourth wall to have interactions with the audience, which can include participation on stage, voting on play topics, discussions and question and answer sections.

Tracy Irish of the Royal Shakespeare Company, who attended The Merchant of Venice Session, described it as "A three-dimensional essay of Shakespeare's plays" (Ní Bhriain 2012: 21). This interactive engagement with Shakespeare's works helps our young audience's:

...make sense of complex language and themes in a post-modern presentation that is enjoyable and aids the learner in making connections between text and performance. (ibid.)

We have been pleased that our audiences have responded enthusiastically to our approach. This support has helped us grow from a very modest audience base to become the main provider of Shakespeare plays for secondary schools in Ireland, with one out of two schools booking our shows year on year. That is, until mid-March 2020, when operations came to an abrupt halt due to the Covid pandemic.

Since then, like many others in the theatre sector, we have had to re-think what we do. For us the best option was to take the Shakespeare Sessions online. However, we didn't come to this decision overnight as the long-term impact of the Covid pandemic had yet to be felt. 


\section{Covid concerns}

In the Summer of 2020, as the transmission rates of Covid-19 were still low, most theatre venues in Ireland were assuming they would be open by September 2020. Theatre directors and managers were asking us if we were still going ahead with our touring plans. However, based on news reports and observations by scientists, it did not look like schools would be back to any kind of normality by September. Hence, we didn't expect to be touring in Autumn 2020 to venues or schools but we weren't 100\% sure. Also, there was the January-April 2021 tour to consider. This was normally the busiest time of the year for us, but would anything be realistically going before live audiences then? Would it be a wiser decision, on our part, to consider creating online content for the 2020-21 school year?

A major consideration in contemplating going online was ascertaining how much teachers would be willing to pay for content. There is so much free Shakespeare content available on the web that we knew we would need to offer something that teachers couldn't get elsewhere. Would they be willing to pay for online content if it was, like our plays, live and interactive? If yes, how much?

To answer questions like these, we sent a three-part survey to 2,646 teachers in our database. Given that it was the middle of summer, the response rate was low but, all the same, we were delighted that 5\% (126 teachers) were able to respond. Their responses are represented in the three graphics following.

A - Thinking about Shakespeare Sessions options for students in November please rank, in order of likelihood, your school's participation in each of the following scenarios:

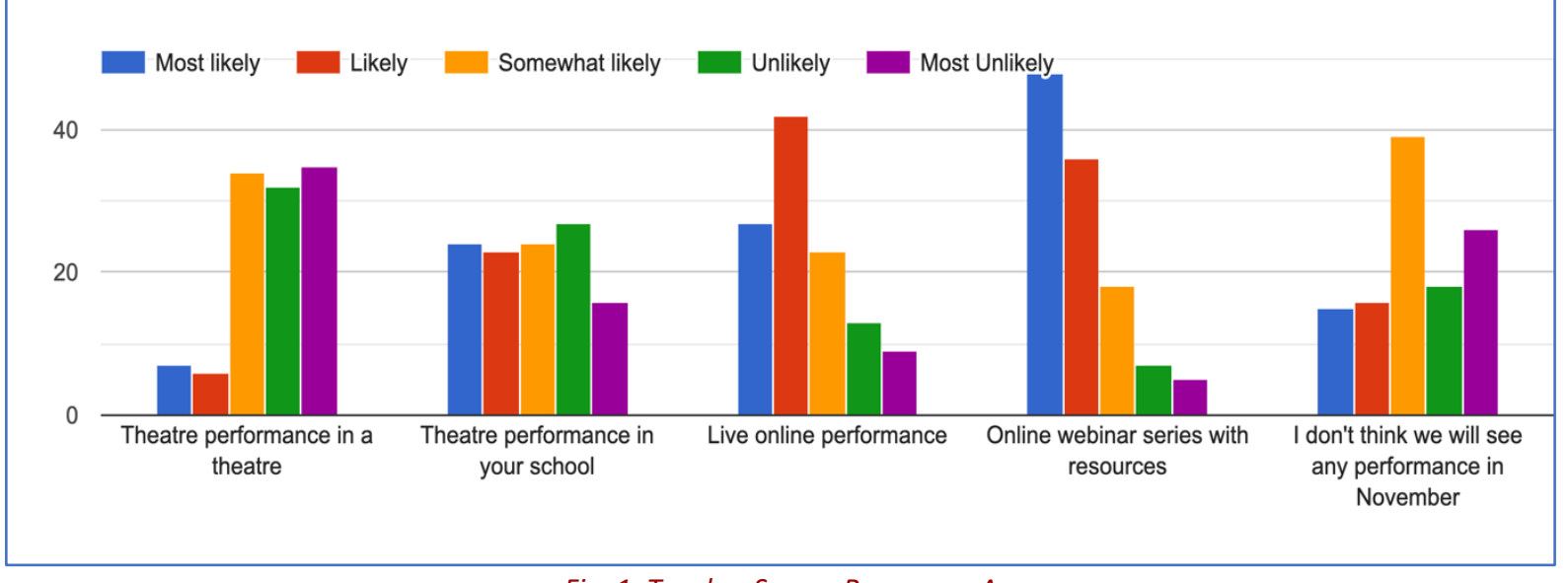

Fig. 1: Teacher Survey Responses $A$ 


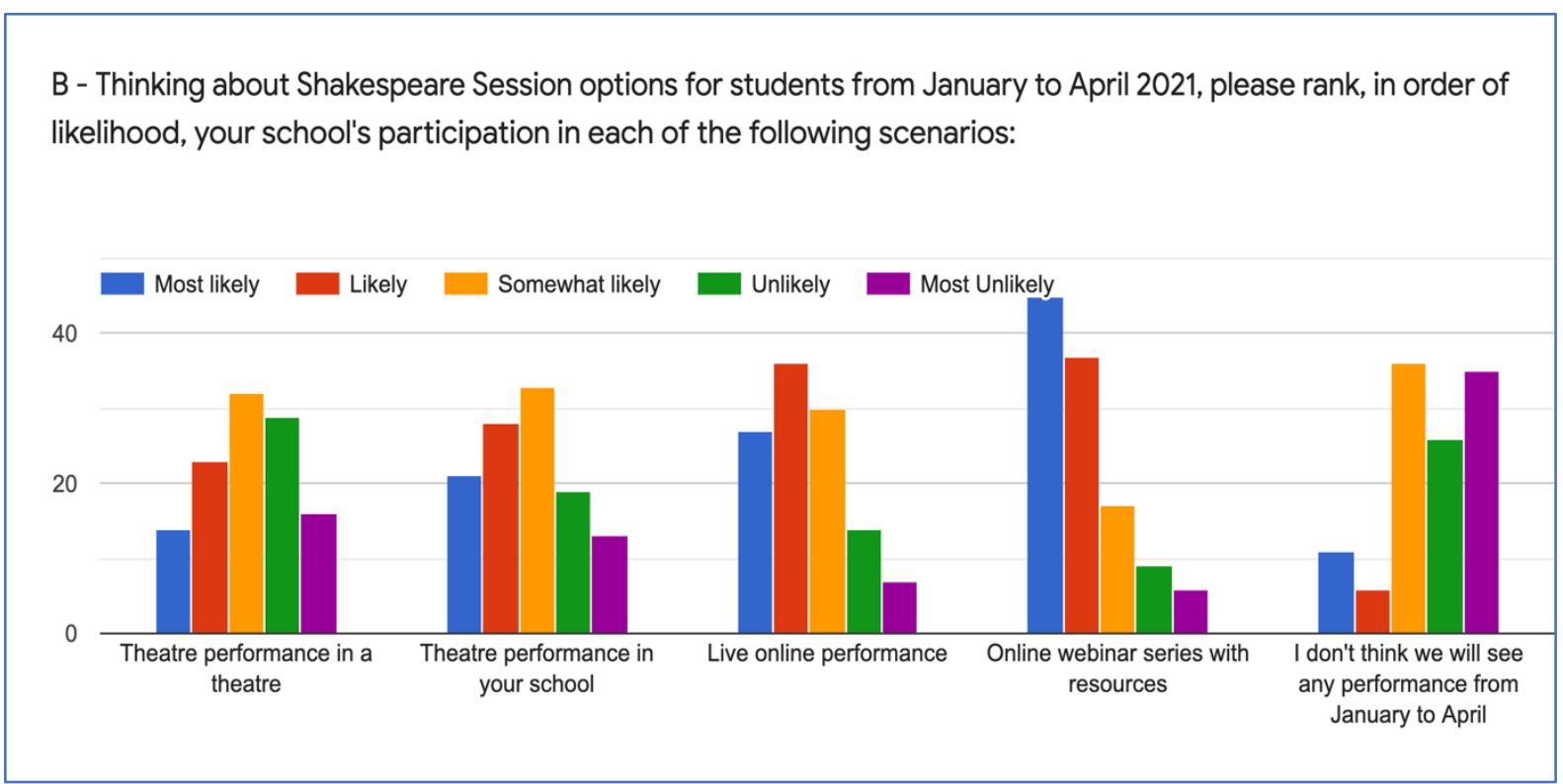

Fig. 2: Teacher Survey Responses B

You will note in Figure 1, that there is very little expectation of attending live theatre in November but for the January to April period as shown in Figure 2, there was more of an expectation that this could happen. However, for both our touring periods in the 2020/21 school year, there was more teacher support for a 'Live online performance' or an 'Online webinar series with resources'.

How much we might be able to charge was answered in the third part of our survey and is represented in Figure 3 below.

C - What would be the maximum price you would consider fair to pay to see an online performance? (for reference our normal ticket price last year was €15)

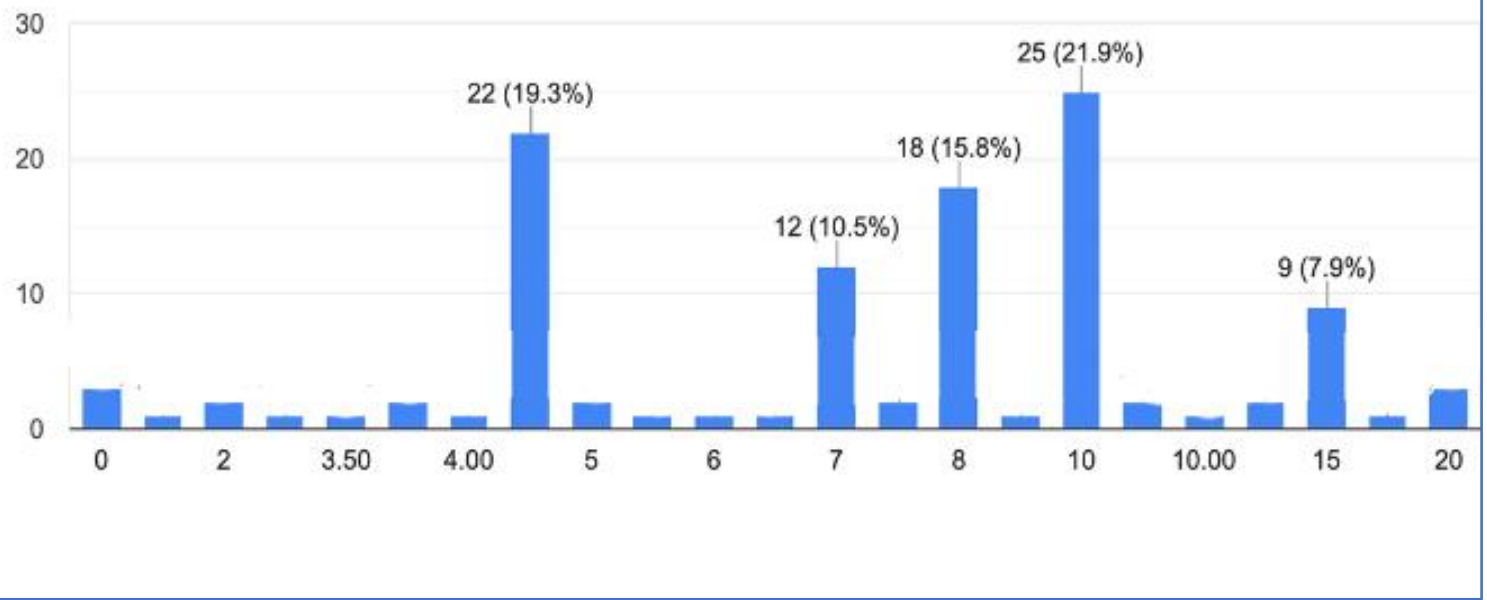

Fig. 3: What price is fair to pay for online content? 
As you can see in the graph above, the majority of teachers were willing to pay between $€ 4.50$ and $€ 10.00$ with about $50 \%$ willing to pay between $€ 7.00-10.00$. So, based on this, and a review of estimated costs, we determined that a price of $€ 9$ per ticket for a live, online webinar would be a reasonable charge per student with further discounts for group bookings. We estimated that as long as we sold about 2,000 tickets per show, we would cover our expenses. Following the survey results, we contacted the 126 teachers who answered it and asked them if they would willing to participate in a live discussion, through Zoom, to give us feedback. Again, it was summer holidays, and responses were few, but six teachers agreed to meet us online to discuss our ideas.

While the teachers who attended the meeting were very enthusiastic and supportive about our plans to make the Shakespeare Sessions live and interactive online, a few expressed concerns over the use of Zoom. Shortly after the lockdown in March 2020, teachers had been advised by the Department of Education and Skills to use Microsoft Teams and not Zoom due to a problem know as: "Zoombombing".

\section{The Zoombomb}

Zoombombing was an incident where hackers were able to enter a meeting without a password and hijack the session by inserting material that was lewd, obscene, racist or homophobic (Billings 2020, Peters 2020). Due to the pandemic, the need for virtual meeting software ballooned and Zoom's platform was overloaded by a huge influx of new users. The Zoom programmers had not anticipated their platform would be used in such an ignorant fashion as Zoombomber hackers did. So, to counter Zoombombing, they updated their platform with a more visible security icon for users with suppression of meeting ID numbers and a change in the default settings to require passwords and waiting rooms for sessions (Zoom Blog 2020).

We had been unaware of this issue but due to the teacher concerns about Zoom (ironically discussed with us while on Zoom), Marcus Bale now looked more closely at Microsoft Teams to compare the competing software's functionality.

The more Marcus learned about Teams, the more he argued that we should use Zoom. Firstly, Zoom allowed users to upload custom virtual graphics which would be useful in our Sessions to place scenes in different locations (Teams now has this feature but not at the time of our consideration). Secondly, for webinars such as the one we were envisaging, you can have videos playing in the background of the Zoom meetings which can also be used for a variety of artistic and educational purposes whereas Teams does not have this facility. Thirdly, at this point in time, we were planning live interactive webinars with teachers acting as the 
intermediary for compiling student votes on character motivation, discussions on themes and question and answer sections. However, if another lockdown was enacted and students were viewing the Shakespeare Session at home, teachers would not have full control of student interaction. Hence, students could potentially turn on video and audio from their sides if the webinar host did not have the ability to stop this from their side within the program settings. Such a feature was necessary for Child Protection and General Data Protection Regulation (GDPR). Luckily, this feature is easily enabled with the Zoom webinar-add-on which allows the meeting to become a webinar while disabling video and audio on the receiver's end. This means students would be able to see and hear us but there would be no possibility for them to be seen or heard by us. However, Teams required a very complicated, not very user-friendly work-around to achieve this function. Thus, the decision was made to use Zoom over Teams. However, there was still the teachers concerns about Zoombombing to allay.

As part of our marketing campaign for The Shakespeare Sessions Online, we set about informing teachers, via e-flyers for the Shakespeare Sessions webinars, how the Zoombombing issue had been resolved and that Zoom was, in most respects, the best platform for our webinars. With this key decision made, we then focussed on content.

\section{Adaptations}

We decided that the first play to receive the online treatment would be 'Romeo and Juliet'. This is the most popular work studied at Junior Cycle level in Ireland (students at this level are generally in the 14-15 years age range) and therefore had the best chance of gaining an online audience.

As our vision and strategy for online content developed, we decided that we didn't want to replace our live productions altogether but create something that would serve as an additional resource for our live productions, which then could be continued to be used when concerns about the Covid pandemic were no longer part of our lives. Based on this remit, Peadar Donohoe wrote the webinars so that they would have a weighted content focus on themes, exam questions and discussions along with key scenes filmed.

Transposing the elements of the live Shakespeare Sessions to the online medium took a considerable amount of work. There were many content and technical elements to be reviewed and actioned such as breaking up a Shakespeare Session script into three schoolperiod time-friendly webinars of 40 minutes each, familiarizing ourselves with various pieces of software such as Canva, Toonly and iMovie, casting the actors, rehearsing, filming, editing, and creating resources handouts to support each webinar. It was hard work and very stressful but by October, 2020 it looked like everything was coming together. 
Then another problem raised its head, as we were trial running a live version of our online Romeo and Juliet Session, our internet connection dropped and we had a weak signal for about an hour. Our provider couldn't tell us the reason. This was of deep concern. Our technical setup was fairly complicated, with not only live discussions, but filmed performances of scenes, audio visuals, slides on themes and animations. Hence, we needed strong stable bandwidth to realise our vision for the Romeo and Juliet Session Online but at the same time we had to rely on an internet connection that could drop at any moment. Hence, the decision had to be made to discard the live option and instead record the whole webinar, including the interactive elements.

\section{Interactive recordings}

You may wonder how could we record a webinar and be interactive with students at the same time. The key to including interaction in a recorded performance was in the resource handouts sent to teachers and students before the recording of each webinar. Each handout included a section called the "Audience Interaction Sheet" which had a number of questions for teachers to pose their students (see Fig. 4).

Teachers were directed to work with their students to answer questions posed on the resource handout. They were given a deadline date to return their answers via email which was a few days before the recording of the webinar. Then their answers, questions and reflections could be included in the webinar.

As complicated at this may seem it has worked out very well and in November 2020 our first Romeo and Juliet Sessions Online went out to schools. Since then, we haven't been Zoombombed and not one teacher has expressed any concerns about using the software. In addition, feedback has been very positive as you can see in the example following:

Webinar 1 is a creative and fun way to revise the play Romeo and Juliet. It presents a mix of performance with stop and think moments. The addition of the interactive material previously gathered from participating students is a strength of the format as it allows students to share their insights and hear the contributions from other schools. Thank you Cyclone for this engaging and effective resource (Gill Sullivan, Our Lady's College, Co. Louth, Ireland. Quote used with permission). 


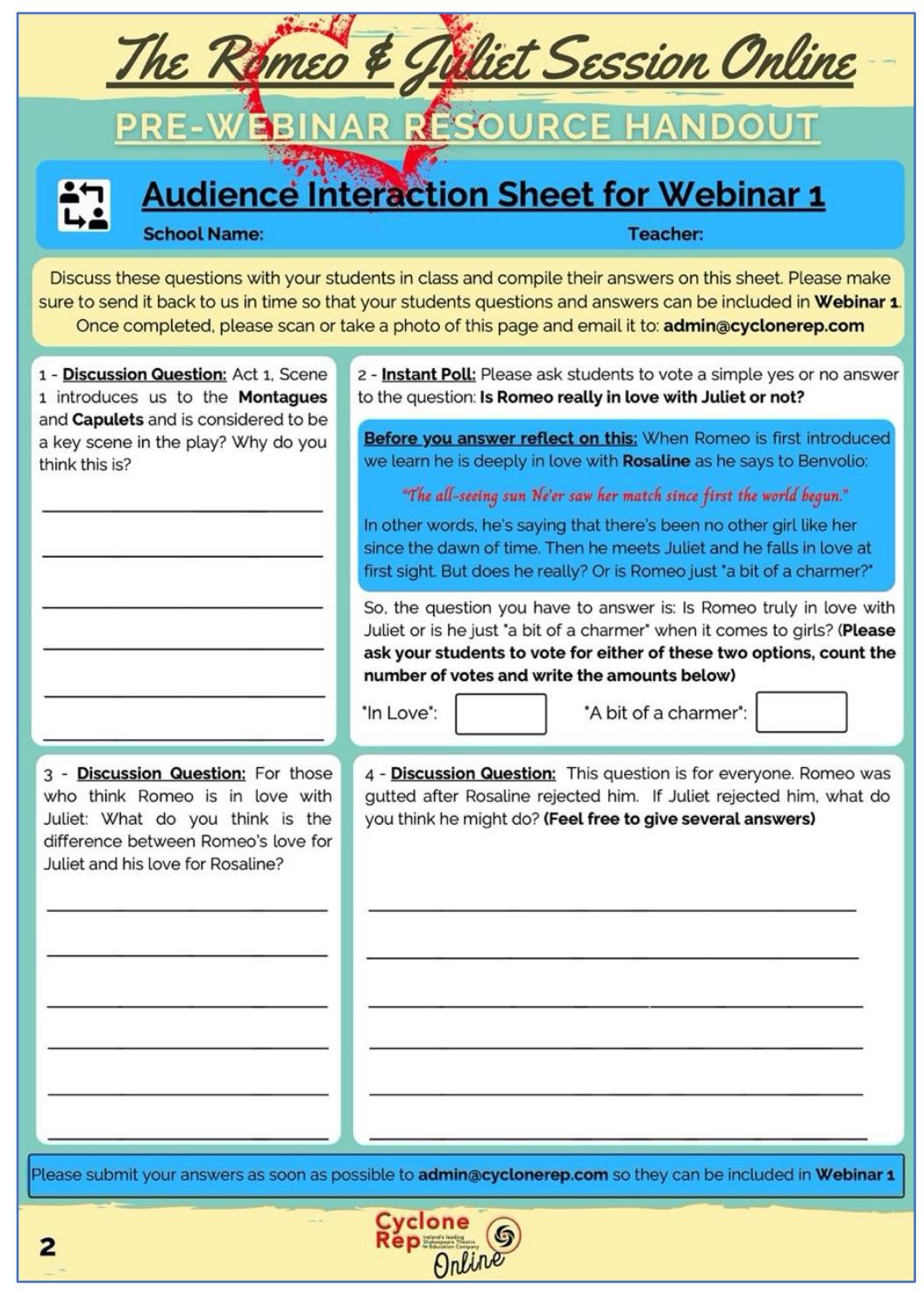

Fig. 4: Audience Interaction Sheet

As word has spread about The Romeo and Juliet Session Online, demand has been brisk with even more schools booked for The King Lear Session Online, which will be available from March 2021.

On an even brighter note, in mid-December 2020 we received a substantial grant from The Arts Council of Ireland which will allow us to hire the services of filmmaker Darren O'Mahony to make high quality recordings of the highlighted scenes from both Romeo and Juliet and King Lear. We are delighted to have a filmmaker join the team to help us add a professional polish to our webinar productions.

If you follow this link https://vimeo.com/499271754/de0d2bcb55, you will get a snapshot of how our online sessions work. If you want more information, feel free to contact us via the Cyclone Rep website at www.cyclonerep.com. 
Donohoe, Bale: To Zoom or not to Zoom

Bibliography

Billings, Kevin (2020): Zoom Announces New Security Changes In Response To Hacks And 'ZoomBombing' Incidents. International Business Times. https://www.ibtimes.com/zoom-announcesnew-security-changes-response-hacks-zoom-bombing-incidents-2955269 [last accessed:

December 22, 2020\}

Ni Bhriain, Seona (2012): Review of theatrical presentations of curriculum plays for second-level school audiences.

http://www.artscouncil.ie/uploadedFiles/wwwartscouncilie/Content/Research and Publications/ Young people children and the arts/Review\%20of\%20theatrical\%20presentations\%20of\%20cu rriculum\%20plays\%20for\%20second-level\%20school\%20audiences.pdf [last accessed: December $21,2020\}$

Peters, Jay (2020): Zoom adds new security and privacy measures to prevent Zoombombing". The Verge. https://www.theverge.com/2020/4/3 /21207643/zoom-security-privacy-zoombombingpasswords-waiting-rooms-default [last accessed: April 12, 2020]

Zoom Blog (2020): How to Keep the Party Crashers from Crashing Your Zoom Event. Zoom Blog. March 20, 2020. https://blog.zoom.us/keep-uninvited-guests-out-of-your-zoom-event [last accessed: January 11, 2021] 